\title{
A Mobile Differentiated Services QoS Model
}

\author{
Jörg Diederich, Lars Wolf \\ Institute of Operating Systems \\ and Computer Networks \\ Technical University of Braunschweig \\ Email: \{dieder|wolf\}@ibr.cs.tu-bs.de
}

\author{
Martina Zitterbart \\ Institute of Telematics \\ University of Karlsruhe (T.H.) \\ Email: zit@tm.uka.de
}

\begin{abstract}
UMTS networks will be based on the Internet Protocol (IP) to provide an efficient support for applications with bursty traffic characteristics, e.g., WWW browsers. Such IP-based networks must include Quality of Service (QoS) mechanisms to enable the usage of real-time applications, such as mobile telephony. Different network services are necessary to satisfy the different needs of applications in wireless mobile networks.

To overcome this problem, this article proposes the Mobile Differentiated Services QoS Model (MoDiQ), a framework to provide QoS in wireless mobile networks. MoDiQ is based on the Differentiated Services approach, developed by the Internet Engineering Task Force for wireline networks. An important part of MoDiQ is the MoDiQ service model which comprises separate services for mobile terminals and non-mobile terminals. This can enhance the efficiency of resource utilization in scenarios where not all mobile terminals are actually moving.
\end{abstract}

\section{Introduction}

A recent trend in mobile communication is the integration of the Internet Protocol (IP) into upcoming mobile networks, e.g., the Universal Mobile Telecommunications System (UMTS). To fulfill the requirements of real-time applications, IP-based networks must provide certain assurances on the IP Quality of Service (QoS) parameters such as bandwidth, delay, delay jitter, and packet loss. Current speech coders, for example, require a minimum bandwidth of $4.75 \mathrm{kbit} / \mathrm{s}$ for an acceptable speech quality of a telephony session [2].

One of the main differences of cellular mobile networks compared to wireline networks is that a mobile terminal can change its point of attachment to the network during an ongoing communication session. This phenomenon, known as a handoff, can lead to a resource shortage which means that the negotiated bandwidth for a session is no longer available after a handoff. In such a case of handoff resource shortage, the communication session must be terminated. This constitutes a major problem because the user of a mobile network, in general, 
expects a QoS-enabled application to work stable over the lifetime of the communication session. An example for a handoff resource shortage in a cellular mobile network is shown in Figure 1. The mobile terminal M has successfully

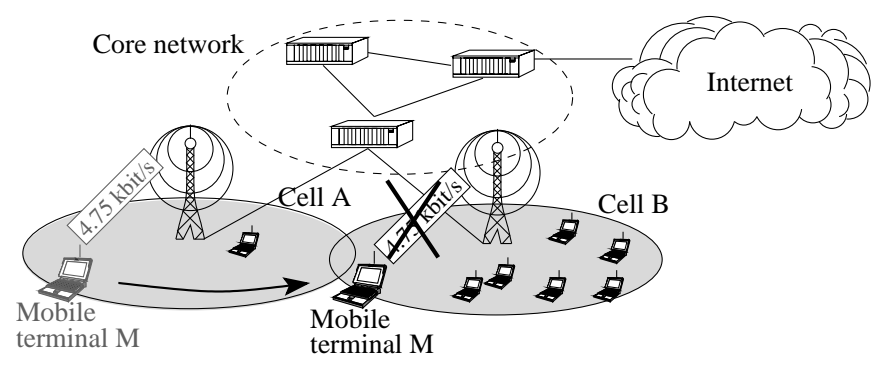

Figure 1: Handoff resource shortage in a cellular mobile network

requested a telephony session with a bandwidth of $4.75 \mathrm{kbit} / \mathrm{s}$ from the mobile network provider and initially resides in the lightly loaded cell $\mathrm{A}$. If this mobile terminal $\mathrm{M}$ needs to perform a handoff to the heavily loaded cell $\mathrm{B}$, a handoff resource shortage occurs because the available bandwidth in cell B is completely utilized by other mobile terminals.

To accommodate handoff resource shortages, a mobility-specific QoS parameter must be considered: the handoff success probability. Providing assurances on the handoff success probability is crucial, especially for future cellular mobile networks where the cell size is decreased to accommodate more mobile terminals in a given geographical area. In this case, the number of handoffs per session and, thus, the probability for a handoff resource shortage can become high even if the mobile terminal moves with only a moderate speed.

Furthermore, a specific service model for mobile networks is needed which comprises the services, provided by the network, and the corresponding QoS parameters. It determines what kind of applications the network can support and which QoS parameters are available for configuration by the service user. An example for service is the Premium Service, known from the Differentiated Services (DiffServ) approach [14. Its characteristics are similar to a leased line, i.e., the data is forwarded with low delay, low delay jitter, and with almost no packet loss. The only QoS parameter to be negotiated between the service user and the service provider is the peak rate of the data stream.

Hence, a framework to provide QoS in wireless mobile networks, the so-called Mobile Differentiated Services QoS Model (MoDiQ) 7], is proposed, comprising:

- A data forwarding plane based on the Differentiated Services approach.

- A control plane providing assurances on the handoff success probability.

- A service model especially suited for usage in wireless mobile networks.

This paper is focused on the MoDiQ service model which extends the legacy DiffServ service model [19] to accommodate the specifics of mobile networks. 
This paper is organized as follows: Section 2 lists the requirements on a service model to be applicable in future mobile networks. The MoDiQ service model is described in detail in Section 3 , followed by a brief simulative evaluation in Section 4 . Section 5 concludes the paper and outlines future work.

\section{Requirements on Service Models in Mobile Networks}

A service model interfaces the QoS-enabled network to the QoS-enabled application. Those QoS parameters which the service model allows to configure, determine how the application can make use of each particular service within the service model. Thus, the usability of a service model is mostly determined by its simplicity [8, 15]. Although a service model should include few services only for simplicity reasons, it must support typical applications of today's networks.

Handoff constitutes a major difference between wireline networks and mobile networks with regard to QoS. Since most real-time applications require a minimum bandwidth, the usage of application adaptation alone is not sufficient to handle QoS variations [5]. The network has to provide QoS assurances with regard to the minimum requirement even in case of adaptive applications in order to support seamless mobile communication. Reserving resources exclusively for handoffs is a well-known method to provide assurances on the handoff success probability [11, 6. The basic drawback is a lower efficiency of network utilization compared to networks without such a handoff resource reservation. Thus, it is important to provide assurances on the handoff success probability only if necessary, i.e., for those mobile terminals which really perform handoff. Therefore, a service model suited for mobile networks should provide separate services for mobile terminals, which potentially perform handoff, and portable terminals which do not perform handoff [13. This way, the efficiency of resource utilization can be significantly increased.

In summary, simplicity, support for typical applications, efficiency, support for assurances on handoff success probabilities, and providing separate services for mobile/portable terminals are considered the most important requirements on service models in mobile networks.

Available proposals for service models have at least one of the following problems when used for wireless mobile networks:

- They comprise many configurable QoS parameters which makes the configuration of a communication session difficult, such as in the Integrated Services approach [5].

- They do not support the above mentioned typical applications properly. For example, services with only qualitative service assurances 15 are not able to provide assurances on the minimum bandwidth.

- They provide no assurances on the handoff success probability.

- They do not distinguish non-mobile terminals, which do not perform handoff, and mobile terminals to increase the efficiency of resource utilization. 


\section{The MoDiQ Service Model}

The MoDiQ service model extends the legacy DiffServ service model, which comprises Premium Service, Olympic Service, and the traditional Best-Effort Service, from three to six service classes as depicted in Figure 2.

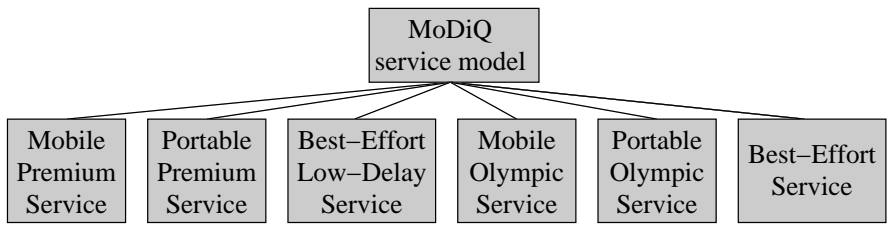

Figure 2: The MoDiQ service model

The legacy DiffServ service model considers only QoS sensitivity in general and delay sensitivity as a subclass of QoS sensitivity (cf., Fig. 3) (a):

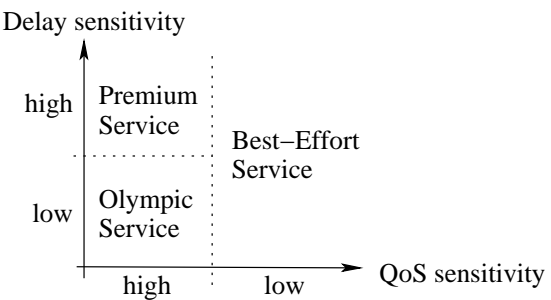

(a) Support for delay/QoS sensitivity

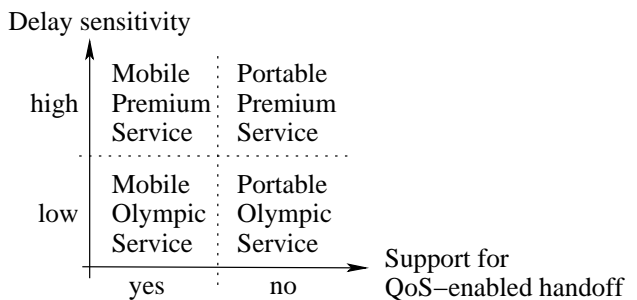

(b) Support for QoS-enabled handoff

Figure 3: Legacy DiffServ service model vs. MoDiQ

The Best-Effort Service is targeted at elastic applications, Premium Service [19] at delay-sensitive real-time applications, and Olympic Service [10] at delay-insensitive applications with minimal bandwidth demands.

The MoDiQ service model adds support for assurances on the handoff success probability for both, Premium Service and Olympic Service. Furthermore, separate services without such an assurance are available for portable terminals (cf., Fig. 3) (b) to increase the efficiency of resource utilization. For this reason, the legacy Premium Service class is divided into two parts: Mobile Premium Service provides low-delay packet delivery with support for assurances on the handoff success probability whereas the Portable Premium Service is a low-delay service with no such support. Analogously, the Olympic Service class is split into a Mobile Olympic Service and a Portable Olympic Service. It is reasonable to provide such a service on the network layer and not only on the wireless link, because a handoff resource shortage might also occur within the wired part of 
the mobile network, for example, on the link from the base station towards the backbone.

The MoDiQ service model further supports loss-sensitive and loss-insensitive applications for both types of applications, those with a high delay sensitivity and those with a low delay sensitivity (cf., Fig. 4).

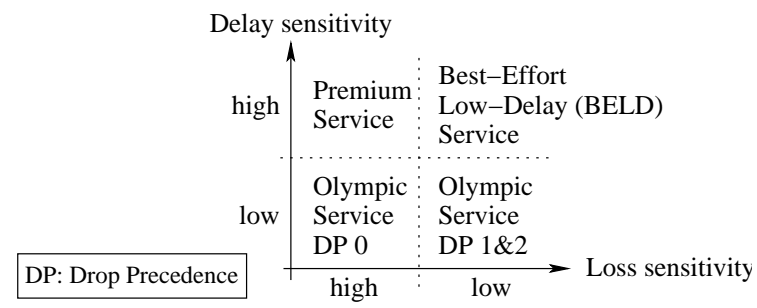

Figure 4: The MoDiQ service model with support for loss-sensitivity

It adds the micro-flow prioritization approach to the Olympic Service to support different degrees of loss-sensitivity for less delay-sensitive applications. This way, a relative service differentiation is introduced within each Olympic Service class. This is useful for QoS-sensitive applications with several micro-flows since they can indicate that one flow (e.g., a video stream) has a lower importance compared to other flows (e.g., an audio stream) 4]. The technical base for this approach is already available. In Olympic Service the drop precedence level can be set in the DiffServ codepoint of each packet to signal the priority of a packet to intermediate nodes. However, the basic intention of Olympic Service is that traffic conditioning decides about the drop precedence level, not the application or the user, so the legacy DiffServ service model provides no means to specify the relative priority for a flow. Therefore, the MoDiQ service model includes an optional specification of a relative priority for a session as QoS parameter for Olympic Service. In this case, the higher drop precedences of Olympic Service can be viewed as a service with packet loss while the lowest drop precedence constitutes a service (almost) without any packet loss.

For delay-sensitive and loss-tolerant applications, there is currently no service available in the legacy DiffServ service model. Thus, the MoDiQ service model contains a third low-delay service called Best-Effort Low-Delay (BELD) Service. This service can take favor of unused Premium Service resources to increase the network utilization. Resource sharing is, therefore, an inherent property of this service.

\subsection{Portable Premium Service}

Similar to Premium Service, Portable Premium Service has a low-delay, lowjitter, low-loss, and assured-bandwidth property. It has a single configurable QoS parameter 'peak-rate' which the service user must configure on a session request. Portable Premium Service is intended for portable terminals which are not forced 
to do handoffs due to mobility while being connected to the network. Such terminals do not need assurances on the handoff success probability, meaning that the service scope is intra-cell.

In contrast to the legacy Premium Service, the special characteristics of the wireless link have to be taken into account for Portable Premium Service [18. Error control schemes can reduce the high bit error rate of wireless links, but they lead to a higher delay or a higher delay jitter compared to wireline networks. Thus, for both the low-delay and the low-loss service characteristics, the meaning of 'low' will be different, i.e., numerically higher, as for the legacy Premium Service. For example, in UMTS the lowest possible delay is planned to be within the range of $80 \mathrm{~ms}$ for the radio access network [1. This article assumes that both, the residual bit error rate and the delay, are small enough for real-time applications using Portable Premium Service.

As an example, a high-end IP-based mobile telephony application without handoff support can utilize Portable Premium Service. To accommodate the wireless link characteristics, the speech data can be coded with speech codecs that can tolerate a low percentage for the residual bit error rate without compromising the acoustic speech comprehensibility significantly (although the speech quality degrades) 16 .

\subsection{Mobile Premium Service}

Mobile Premium Service is an enhanced Portable Premium Service where the service scope is not limited to a single cell. It provides assurances on the handoff success probability, i.e., the amount of bandwidth assigned in the old cell will be available in the new cell with a certain probability. However, this is only possible if the handoff is performed between two cells of the same radio access network (RAN), a so-called intra-RAN handoff. An example application for Mobile Premium Service is a high-end mobile telephony application with intraRAN handoff support.

Mobile Premium Service can not deal with inter-RAN handoff because of the significantly different availability of resources in different radio access networks. For example, the available resources per terminal in wideband mobile networks such as Wireless LANs are up to $54 \mathrm{Mbit} / \mathrm{s}$ in an empty cell. In narrow-band networks, such as GPRS, the available bandwidth per mobile terminal is theoretically up to $171 \mathrm{kbit} / \mathrm{s}$, but on average only $40 \mathrm{kbit} / \mathrm{s}$. Thus, providing assurances on the handoff success probability for a handoff from Wireless LAN to GPRS is almost impossible (although it may work in the opposite direction) for those session with a high bandwidth requirement. Therefore, Mobile Premium Service can only be provided for an intra-RAN scope.

Furthermore, admission control considers not only the resource situation in the current cell as for Portable Premium Service, but takes also the resource situation in the neighboring cells into account to provide assurances on the handoff success probabilities. This so-called distributed admission control is necessary because local schemes cannot give a sufficiently high assurance on the handoff success probability. 


\section{Portable Premium Service vs. Mobile Premium Service}

The advantage of Portable Premium Service is that its users have a higher probability of being admitted in a mobile network with many mobile terminals, a high number of prioritized handoffs and with cells with different resource utilization levels. As an example, consider a cell X serving a city highway and a neighboring cell $\mathrm{Y}$ from which the highway can be reached and which also serves some apartments (cf., Fig. 5). If the 'highway cell $\mathrm{X}$ ' is highly loaded and cell $\mathrm{Y}$ is

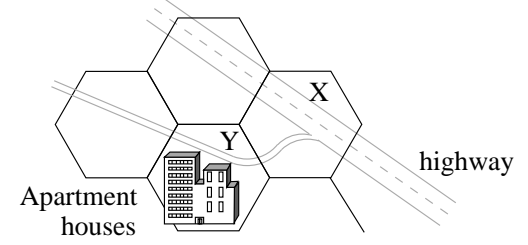

Figure 5: Example: Apartments in the neighborhood of a highway

only lightly loaded, Mobile Premium Service requests in cell Y are denied owing to the distributed admission control algorithm of Mobile Premium Service: It considers not only the resource situation in the current cell, but takes also the resource situation in the neighboring cells into account to provide a sufficiently high assurance on the handoff success probabilities. This is necessary to block those sessions which will enter the highway cell $\mathrm{X}$ and, hence, experience handoff drop with a high probability. However, portable users of the mobile network in the apartments do not want to move to the highway cell X. Therefore, Portable Premium Service enables these users to receive resources which increases the resource utilization level of low-delay resources in cell Y.

\subsection{Best-Effort Low-Delay Service}

Similar to Portable Premium Service and Mobile Premium Service, Best-Effort Low-Delay (BELD) Service belongs to the low-delay services. In contrast to the former two low-delay services, packet drops can occur in BELD Service for a certain period of time. The introduction of BELD Service has two objectives:

1. It utilizes unused Premium Service resources (i.e., Portable and Mobile) to increase the efficiency in the utilization. These resources are assumed to provide a considerable part of the operator's revenue.

2. It provides a low-delay, but lossy service for delay-sensitive and loss-tolerant applications. Since all low-delay applications can naturally work using Premium Service, BELD Service must be significantly cheaper to attract the interest of potential customers.

For example, a low-cost mobile telephony application based on a loss-tolerant speech codec may make use of the BELD Service. The users of such an application 
may accept the varying speech quality and occasional service disruptions because of a lower price compared to the high-end Mobile Premium Service. Such a lowcost service is interesting, for example, for parents to provide a cheap mobile telephony service to their children.

BELD Service traffic is intended to utilize unused Premium Service resources to achieve a low-delay characteristic. These resources are not utilized up to $100 \%$ in general for the following reasons:

1. To achieve a high assurance on the handoff success probability even for traffic patterns which have a low probability to occur, the necessary handoff resources to reserve are estimated rather conservatively in MoDiQ. Thus, some handoff resources are available for BELD Service on average, i.e., if the mobility pattern does not represent a worst-case scenario so that less handoff resources are necessary than expected.

2. The negotiated Portable/Mobile Premium Service peak rate for a single flow is rarely used up to $100 \%$. For example, silence suppression can reduce the average necessary bandwidth of a telephony application to about 30$50 \%$ compared to a speech codec without silence suppression. However, it is not possible to increase the utilization by subscribing to a lower rate than the peak rate of the codec. This would lead to substantial service disruptions in case of long speech periods which is against the 'low loss' property of such a 'Premium Service'.

\subsection{Portable/Mobile Olympic Service \& Micro-Flow Prioritization}

In contrast to Premium Service, Olympic Service is intended to support service differentiation for bursty data flows. It provides an assurance on the negotiated minimal bandwidth but no assurances on delay or jitter. Thus, it is well-suited, for example, for streaming applications, which require a certain assurance on a minimal bandwidth and which can compensate a varying delay to a certain extent with buffers at the receiver.

Similar to the differentiation Portable/Mobile Premium Service, Olympic Service is divided into Portable Olympic Service and Mobile Olympic Service in the MoDiQ service model. However, the assurance on the handoff success probability in Mobile Olympic Service is only valid for the traffic which is within the negotiated minimal rate. One of the remaining problems in mobile networks is that the available bandwidth may vary heavily, for example, at inter-RAN handoffs. Adding the micro-flow prioritization scheme to Olympic Service, as proposed in the MoDiQ service model, can improve the overall QoS for applications with several micro-flows. The service user can signal the priority of a flow to the first boundary node using the DiffServ codepoint. This implicit signaling mechanism is scalable since a multi-field classification on interior nodes is not necessary to identify those flows which should be affected by packet loss preferentially. This way, user-provided priorities for the micro-flows become possible [4. 


\section{Simulations}

MoDiQ has been evaluated extensively using the network simulator ns2. To show the advantage of having separate services for portable terminals and mobile terminals, this section examines the gain of using Portable Premium Service in addition to Mobile Premium Service.

\subsection{Simulation Model}

The network model consists of two parts. The wireless part is composed of nine base stations which are placed onto a rectangular grid. The distance between two base stations is $700 \mathrm{~m}$ horizontally and vertically which is a typical distance for mobile networks in a densely populated city area. The cell size is $800 \mathrm{~m}$ so the coverage areas of two neighboring base stations overlap up to $100 \mathrm{~m}$ to enable soft handoffs without interruptions of connectivity. The handoff control algorithm is based on a hysteresis [17. which can avoid subsequent handoffs between two base stations within a short period of time (the so-called flip-flop effect). The wireless network is based on the IEEE 802.11 standard to simulate realistic effects such as collisions on the air interface since it has been found to be important to model these effects to achieve realistic simulation results 9 . The base stations are interconnected via a tree-like topology (cf., Fig. 6) leading towards the root node of the backbone which itself may be connected to the Internet in a realworld scenario. The node connected to the root-node represents a node in the

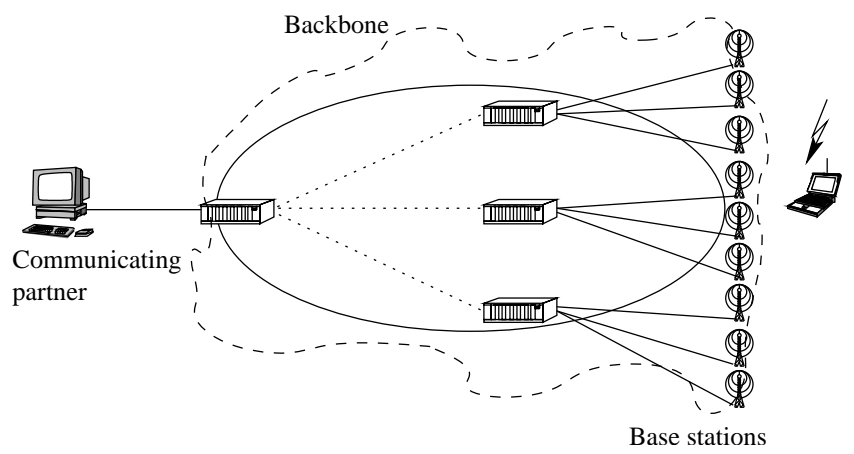

Figure 6: The network model

Internet and is the communicating partner of all mobile terminals.

The network is designed such that each base station can carry up to 100 sessions simultaneously. The session duration is modeled as an exponential distribution with a mean of $180 \mathrm{~s}$, which models telephony sessions quite accurately [3].

As mobility model, the so-called Random-Move scheme is used. It consists of a $3 \times 3$ mobility cell scenario (with one base station in the center of each cell) where eight cells are in use (one edge cell is not used to create a higher load in the center cell). In each cell, the probability of a mobile terminal to change to 
one of the neighboring cells is equal. Mobile terminals have the same probability to start in any of these eight cells, the average speed is a truncated Gaussian distribution [17] with different means and a maximum deviation of $\pm 20 \%$. The Random-Move scenario is simulated with an average speed of $17 \mathrm{~m} / \mathrm{s}$ (about $60 \mathrm{~km} / \mathrm{h}$ ), representing scenarios with no traffic jams where the terminals can move fluently, for example, on a main-street with coordinated traffic lights. To show the effect of having portable terminals in the network, only $25 \%$ of the terminals move in this scenario, the remaining $75 \%$ do not move during the simulations (the so-called 'Static Random-Move scenario').

\subsection{Simulation Results}

We propose that Portable Premium Service is useful in areas where cells have different resource utilizations and where new session requests in a cell with free resources are rejected because of a highly loaded neighboring cell. This is true in the Random-Move scenario where the center cell has a higher resource utilization than the surrounding cells.

Figure 7 depicts the gain of introducing Portable Premium Service in the static Random-Move scenario where $75 \%$ of the terminals are non-mobile. In

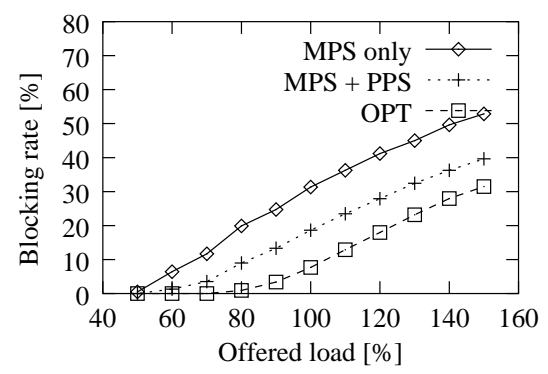

Figure 7: Portable Premium Service simulations: Blocking rate

the Mobile Premium Service simulations ('MPS only' in the figure), portable terminals request Mobile Premium Service whereas they request Portable Premium Service in the simulations with both Mobile Premium Service and Portable Premium Service ('MPS + PPS' in the figure). In the former case, this means that the distributed admission control scheme of Mobile Premium Service is used for all mobile terminals regardlessly whether they perform handoff or not. This is the only difference between the two simulation runs so that it is ensured that a change in the result comes from the introduction of Portable Premium Service. For comparison, the simulation have been performed for a theoretically optimal scheme 12. (OPT in the figure). The blocking rate for the Portable Premium Service simulations, i.e., the probability that a new session request is denied, is much closer to the optimal approach than the blocking rate of the Mobile Premium Service simulations. Depending on the offered load, the introduction of Portable Premium Service increases the number of accepted sessions 
up to $25 \%$. At the same time, no additional handoff resource shortages occur so that no sessions have to be terminated after a handoff. The introduction of Portable Premium Service can, therefore, provide a significant enhancement of the resource utilization.

\section{Summary and Future Work}

The Mobile Differentiated Services QoS model (MoDiQ) is a simple and scalable solution for providing QoS in wireless mobile networks. It enhances the legacy DiffServ service model with support for QoS-enabled handoffs, if necessary, and with separate services for loss-sensitive and loss-tolerant applications. QoS-supported handoffs are supported in Mobile Premium Service and Mobile Olympic Service: These services give assurances on the handoff success probability for delay-sensitive and less delay-sensitive applications, respectively. Providing separate services without support for QoS-enabled handoff (i.e., Portable Premium Service and Portable Olympic Service) can potentially improve the resource utilization. The Best-Effort Low-Delay (BELD) Service is part of the $M o D i Q$ service model for two reasons: To provide a Best-Effort-like service for loss-tolerant delay-sensitive applications, such as low-cost telephony, and to increase the utilization of low-delay resources. Thus, the MoDiQ service model supports important applications in future wireless mobile networks such as mobile telephony, WWW browsing and streaming applications.

Future work includes several areas: The MoDiQ proposal is currently focused on providing assurances on the handoff success probability on the bottleneck links only. These links are presumably the wireless link or the last wired mile, which connects the base station to the backbone of the mobile network. To achieve end-to-end QoS assurances, it is possible to deploy a legacy DiffServ resource management (e.g., a Bandwidth Broker) within the backbone of the mobile network. This requires a coordination of the components of MoDiQ with this backbone resource management. A challenge is to maintain the high scalability of MoDiQ in such an end-to-end scenario.

Further investigations may evaluate the usage of adaptive applications which can work under different resource situations. This way, more sessions can possibly be supported simultaneously and the QoS can be enhanced for adaptive applications if sufficient resources become available.

\section{Acknowledgment}

The authors would like to acknowledge Thorsten Lohmar and Dr. Ralf Keller from Ericsson Eurolab Deutschland GmbH, Aachen, Germany, for their insightful comments and discussions.

\section{References}

[1] QoS Concept and Architecture (Release 1999). Technical report, 3GPP: Technical Specification Group Services and System Aspects, Jan. 2002. 
[2] AMR speech Codec: General description (Release 4). Technical report, 3GPP: Technical Specification Group Services and System Aspects, Apr. 2000.

[3] BAKOM: Bundesamt für Kommunikation, Abteilung Telecomdienste. Average voice call durations in Switzerland (1998-2000). Report on Fernmeldestatistik 2000, Biel, Schweiz, Nov. 2001. In German.

[4] A. Bouch, M. Sasse, and H. DeMeer. Of packets and people: A user-centered approach to Quality of Service. In Proceedings of 9th International conference on Quality of Service (IWQoS'00), Pittsburgh, PA, USA, June 2000.

[5] R. Braden, D. Clark, and S. Shenker. Integrated Services in the Internet Architecture: an Overview. RFC (Informational) 1633, IETF, June 1994.

[6] S. Choi and K. Shin. A comparative study of bandwidth reservation and admission control schemes in QoS-sensitive cellular networks. ACM Wireless Networks, 6(4):289-305, 2000.

[7] J. Diederich, T. Lohmar, M. Zitterbart, and R. Keller. A QoS Model for Differentiated Services in Mobile Wireless Networks. In Digest of the 11th IEEE Workshop on Local and Metropolitan Area Networks (LANMAN 2001). IEEE, Mar. 2001.

[8] P. Ferguson and G. Huston. Quality of Service: Delivering QoS on the Internet and in Corporate Networks. Wiley \& Sons, New York, USA, Jan. 1998.

[9] J. Heidemann, N. Bulusu, J. Elson, C. Intanagonwiwat, K. Lan, Y. Xu, W. Ye, D. Estrin, and R. Govindan. Effects of detail in wireless network simulation. In Proc. of the SCS Multiconference on Distributed Simulation, pages 3-11, Phoenix, USA, Jan. 2001.

[10] J. Heinanen, F. Baker, W. Weiss, and J. Wroclawski. Assured Forwarding PHB Group. RFC (Proposed Standard) 2597, IETF, June 1999.

[11] D. Hong and S. Rappaport. Traffic Model and Performance Analysis for Cellular Mobile Radio Telephone Systems with Prioritized and Nonprioritized Handoff Procedures. IEEE Transactions on Vehicular Technology, 35(3):77-92, Aug. 1986. See also: CEAS Technical Report No. 773, June 1, 1999, College of Engineering and Applied Sciences, State University of New York, Stony Brook, NY 11794, USA.

[12] R. Jain and E. Knightly. A Framework for Design and Evaluation of Admission Control Algorithms in Multi-Service Mobile Networks. In Proc. of the IEEE Conference on Computer Communications (IEEE Infocom), New York, Mar. 1999.

[13] S. Jiang, B. Li, X. Luo, and D. Tsang. A Modified Call Admission Control Scheme and Its Performance. ACM Wireless Networks, 7(2):127-138, Mar. 2001.

[14] K. Nichols, S. Blake, F. Baker, and D. Black. Definition of the Differentiated Services Field (DS Field) in the IPv4 and IPv6 Headers. RFC (Proposed Standard) 2474, IETF, Dec. 1998.

[15] J. Ruutu and K. Kilkki. Simple Integrated Media Access - a Comprehensive Service for Future Internet. In Proc. of the IFIP Conference on Performance of Information and Communications Systems (PICS), Lund, Sweden, May 1998.

[16] L. Sun, G. Wade, B. Lines, and E. Ifeachor. Impact of Packet Loss Location on Perceived Speech Quality. In IP Telephony Workshop, pages 114-122, New York, USA, Apr. 2001.

[17] N. Tripathi, J. Reed, and H. VanLandingham. Handoff in Cellular Systems. IEEE Personal Communications Magazine, 5(6):26-37, Dec. 1998.

[18] A. Veres, M. Barry, L.-H. Sun, and A. Campbell. Supporting Service Differentation in Wireless Packet Networks using Distributed Control. IEEE Journal on Selected Areas in Communications, 19(10):2081-2093, Oct. 2001.

[19] L. Zhang, V. Jacobson, and K. Nichols. A Two-bit Differentiated Services Architecture for the Internet. RFC (Informational) 2638, IETF, July 1999. 